\title{
Transversal Lattices
}

\author{
Joseph E. Bonin \\ Department of Mathematics \\ The George Washington University \\ Washington, D.C. 20052, USA \\ jbonin@gwu.edu
}

Submitted: Sep 26, 2007; Accepted: Jan 7, 2008; Published: Jan 14, 2008

Mathematics Subject Classification: 05B35

\begin{abstract}
A flat of a matroid is cyclic if it is a union of circuits; such flats form a lattice under inclusion and, up to isomorphism, all lattices can be obtained this way. A lattice is a Tr-lattice if all matroids whose lattices of cyclic flats are isomorphic to it are transversal. We investigate some sufficient conditions for a lattice to be a Tr-lattice; a corollary is that distributive lattices of dimension at most two are Tr-lattices. We give a necessary condition: each element in a Tr-lattice has at most two covers. We also give constructions that produce new Tr-lattices from known Tr-lattices.
\end{abstract}

\section{Introduction}

A flat $X$ of a matroid $M$ is cyclic if the restriction $M \mid X$ has no isthmuses. Ordered by inclusion, the cyclic flats form a lattice, which we denote by $\mathcal{Z}(M)$. Every lattice is isomorphic to the lattice of cyclic flats of some (bi-transversal) matroid [4, 8]. (All lattices considered in this paper are finite.) Although $M$ is determined by its cyclic flats and their ranks, in most cases $\mathcal{Z}(M)$, viewed as an abstract lattice, reveals little about $M$. However, for certain lattices $L$, it is shown in $[1,2]$ that if $\mathcal{Z}(M)$ is isomorphic to $L$, then $M$ is transversal; lattices with this property are transversal lattices or Tr-lattices. In [4], lattices of width at most two are shown to be Tr-lattices. In this paper we treat more general sufficient conditions for a lattice to be a Tr-lattice and we prove a necessary condition. More specifically, Section 3 introduces MI-orderable lattices (which include distributive lattices of dimension at most two) and shows they are Tr-lattices; Section 4 shows that each element of a Tr-lattice has at most two covers. Lastly, Section 5 gives ways to construct new MI-orderable lattices (resp., Tr-lattices) from known MI-orderable lattices (resp., Tr-lattices). 


\section{Background}

We assume familiarity with basic matroid theory. Our notation and terminology for matroid theory follow [7]; for ordered sets we mostly follow [10]. For a collection $\mathcal{F}$ of sets, we write $\bigcap(\mathcal{F})$ for the intersection $\bigcap_{X \in \mathcal{F}} X$ and $\bigcup(\mathcal{F})$ for $\bigcup_{X \in \mathcal{F}} X$.

Recall that any ordered set $P$ can be embedded in a product of chains; the dimension of $P$ is the least number of chains for which there is such an embedding. An antichain in $P$ is a collection of mutually incomparable elements of $P$. The width of $P$ is the maximal cardinality among the antichains of $P$. We say $y$ is a cover of $x$ in $P$ if $x<y$ and there is no $z$ in $P$ with $x<z<y$. An ideal in $P$ is a subset $I$ of $P$ such that if $x \in I$ and $y \leq x$, then $y \in I$; dually, $F \subseteq P$ is a filter if whenever $x \in F$ and $y \geq x$, then $y \in F$. The least and greatest elements in a lattice are denoted $\hat{0}$ and $\hat{1}$, respectively. The atoms of a lattice are the elements that cover $\hat{0}$; dually, the coatoms are the elements that $\hat{1}$ covers.

The lattice $\mathcal{Z}(M)$ of cyclic flats of $M$ has the same join operation as the lattice of flats: $A \vee B=\operatorname{cl}(A \cup B)$. In contrast to the lattice of flats, the meet operation of $\mathcal{Z}(M)$ might not be intersection: $X \wedge Y$ is the union of the circuits that are contained in $X \cap Y$.

A matroid on a given set is determined by its collection of cyclic flats and their ranks. In some cases we will ignore the cyclic flats and instead focus on the ranks assigned to the elements of an abstract lattice; this is justified by the following special case of [8, Theorem 1].

Proposition 2.1 Let $L$ be a lattice. Given $\rho: L \rightarrow \mathbb{Z}$ with

(a) $\rho(\hat{0})=0$,

(b) $\rho(x)<\rho(y)$ whenever $x<y$, and

(c) $\rho(x \vee y)+\rho(x \wedge y) \leq \rho(x)+\rho(y)$ whenever $x$ and $y$ are incomparable,

there is a matroid $M$ and an isomorphism $\phi: L \rightarrow \mathcal{Z}(M)$ with $\rho(x)=r(\phi(x))$.

A key result we use to prove that certain lattices are (or are not) Tr-lattices is the following characterization of transversal matroids, which was first formulated by Mason using cyclic sets and later refined to cyclic flats by Ingleton [5]. (The statement in [5] uses all nonempty collections of cyclic flats, but an elementary argument shows that it suffices to consider nonempty antichains of cyclic flats.)

Proposition 2.2 A matroid $M$ is transversal if and only if for every nonempty antichain $\mathcal{A}$ in $\mathcal{Z}(M)$,

$$
r(\bigcap(\mathcal{A})) \leq \sum_{\mathcal{F} \subseteq \mathcal{A}}(-1)^{|\mathcal{F}|+1} r(\bigcup(\mathcal{F})) .
$$

It follows from the description of joins in $\mathcal{Z}(M)$ that the right side in this inequality can be replaced by the corresponding alternating sum of ranks of joins of cyclic flats. 
Since the complements of the flats of a matroid are the unions of its cocircuits, $X$ is a cyclic flat of $M$ if and only if $E(M)-X$ is a cyclic flat of the dual, $M^{*}$. Thus, $\mathcal{Z}\left(M^{*}\right)$ is isomorphic to the order dual of $\mathcal{Z}(M)$.

Let $S$ and $E$ be the least and greatest cyclic flats of $M$. Note that for $X \in \mathcal{Z}(M)$, the lattice $\mathcal{Z}(M \mid X)$ is the interval $[S, X]$ in $\mathcal{Z}(M)$; dually, $\mathcal{Z}(M / X)$ is isomorphic to the interval $[X, E]$ in $\mathcal{Z}(M)$ via the isomorphism $Y \mapsto Y \cup X$. (The lattices of cyclic flats of other minors are not as simple to describe.)

\section{Sufficient conditions for a lattice to be a Tr-lattice}

The main result of this section, Theorem 3.2, implies that if the ordering property in the following definition holds for all antichains of a lattice $L$, then $L$ is a Tr-lattice.

Definition 3.1 For an antichain $\mathcal{A}$ in a lattice $L$, an MI-ordering of $\mathcal{A}$ is a permutation $a_{1}, a_{2}, \ldots, a_{t}$ of $\mathcal{A}$ so that

(i) $a_{i} \vee a_{i+1} \vee \cdots \vee a_{k}=a_{i} \vee a_{k}$ for $1 \leq i<k \leq t$ and

(ii) $\left(a_{1} \wedge a_{2} \wedge \cdots \wedge a_{k}\right) \vee a_{k+1}=a_{k} \vee a_{k+1}$ for $1<k<t$.

An antichain is MI-orderable if it has an MI-ordering. A lattice is MI-orderable if each of its antichains is MI-orderable.

Theorem 3.2 Let $M$ be a matroid.

(i) Each $M I$-orderable antichain in $\mathcal{Z}(M)$ satisfies inequality (MI).

(ii) If $\mathcal{Z}(M)$ is $M I$-orderable, then $M$ and all of its minors are transversal.

We prove Theorem 3.2 via a sequence of lemmas. (The meet and join operations in the next lemma and other results are in $\mathcal{Z}(M)$.)

Lemma 3.3 Let $A_{1}, A_{2}, \ldots, A_{t}$ be an antichain of cyclic flats in a matroid $M$ such that $\left(A_{1} \wedge A_{2} \wedge \cdots \wedge A_{k}\right) \vee A_{k+1}=A_{k} \vee A_{k+1}$ whenever $1 \leq k<t$. Then for $k$ with $k \leq t$,

$$
r\left(A_{1} \cap A_{2} \cap \cdots \cap A_{k}\right) \leq \sum_{i=1}^{k} r\left(A_{i}\right)-\sum_{i=1}^{k-1} r\left(A_{i} \cup A_{i+1}\right) .
$$

Proof. Induct on $k$. Equality holds for $k=1$. For the inductive step, inequality (1) and the semimodular inequality applied to $A_{1} \cap A_{2} \cap \cdots \cap A_{k}$ and $A_{k+1}$ give

$r\left(A_{1} \cap A_{2} \cap \cdots \cap A_{k+1}\right)+r\left(\left(A_{1} \cap A_{2} \cap \cdots \cap A_{k}\right) \cup A_{k+1}\right) \leq \sum_{i=1}^{k+1} r\left(A_{i}\right)-\sum_{i=1}^{k-1} r\left(A_{i} \cup A_{i+1}\right)$,

so if we show $r\left(\left(A_{1} \cap A_{2} \cap \cdots \cap A_{k}\right) \cup A_{k+1}\right)=r\left(A_{k} \cup A_{k+1}\right)$, then the inequality we want follows. This equality holds since $A_{1} \wedge A_{2} \wedge \cdots \wedge A_{k} \subseteq A_{1} \cap A_{2} \cap \cdots \cap A_{k} \subseteq A_{k}$ and $\left(A_{1} \wedge A_{2} \wedge \cdots \wedge A_{k}\right) \vee A_{k+1}=A_{k} \vee A_{k+1}$. 
Lemma 3.4 An antichain $\mathcal{A}$ in $\mathcal{Z}(M)$ satisfies inequality (MI) if it can be ordered as $A_{1}, A_{2}, \ldots, A_{t}$ with

(i) $A_{i} \vee A_{i+1} \vee \cdots \vee A_{k}=A_{i} \vee A_{k}$ whenever $1 \leq i<k \leq t$ and

(ii) $r\left(A_{1} \cap A_{2} \cap \cdots \cap A_{t}\right) \leq \sum_{i=1}^{t} r\left(A_{i}\right)-\sum_{i=1}^{t-1} r\left(A_{i} \cup A_{i+1}\right)$.

Proof. Assume properties (i) and (ii) hold. For $1 \leq i \leq j \leq t$, set

$$
\mathcal{A}_{i, j}=\left\{\mathcal{F}: \mathcal{F} \subseteq \mathcal{A}, i=\min \left(k: A_{k} \in \mathcal{F}\right) \text {, and } j=\max \left(k: A_{k} \in \mathcal{F}\right)\right\} .
$$

Thus, if $\mathcal{F} \in \mathcal{A}_{i, j}$, then $\left.\mathrm{cl}(\bigcup \mathcal{F})\right)=A_{i} \vee A_{j}$. If $j>i+1$, then the terms on the right side of inequality (MI) that arise from the sets in $\mathcal{A}_{i, j}$ cancel since there is a parity-switching involution $\phi$ of $\mathcal{A}_{i, j}$ : fix $k$ with $i<k<j$ and let

$$
\phi(\mathcal{F})= \begin{cases}\mathcal{F} \cup\left\{A_{k}\right\}, & \text { if } A_{k} \notin \mathcal{F} \\ \mathcal{F}-\left\{A_{k}\right\}, & \text { if } A_{k} \in \mathcal{F}\end{cases}
$$

Thus, inequality (MI) reduces to the inequality that is assumed in property (ii).

The lemmas above show that MI-orderable lattices are Tr-lattices. To prove the stronger assertion in part (ii) of Theorem 3.2, we show in Lemma 3.6 that if the antichains in $\mathcal{Z}(M)$ satisfy the hypotheses of Lemma 3.4, then the same is true for any single-element deletion $M \backslash x$ or contraction $M / x$. (Unlike the hypotheses of Theorem 3.2, condition (ii) in Lemma 3.4 is not a lattice-theoretic property.) We will use the following lemma about the cyclic flats of $M \backslash x$ and $M / x$; the statement is evident for $M \backslash x$ and follows for $M / x$ by an elementary duality argument.

Lemma 3.5 For an element $x$ of $M$ and a cyclic flat $A$ of either $M \backslash x$ or $M / x$, the flat $\bar{A}=\operatorname{cl}_{M}(A)$ of $M$ is cyclic; furthermore, $\bar{A}$ is either $A$ or $A \cup x$.

Lemma 3.6 If each antichain in $\mathcal{Z}(M)$ can be ordered so that properties (i) and (ii) of Lemma 3.4 hold, then the same is true for each antichain in $\mathcal{Z}(M \backslash x)$ and each antichain in $\mathcal{Z}(M / x)$.

Proof. We use the notation $\bar{A}$ of Lemma 3.5. Let $\mathcal{A}$ be an antichain in $\mathcal{Z}(M / x)$. Note that $\{\bar{A}: A \in \mathcal{A}\}$ is an antichain in $\mathcal{Z}(M)$. By hypothesis, there is an ordering $A_{1}, A_{2}, \ldots, A_{t}$ of $\mathcal{A}$ so that the following properties hold:

$$
\begin{gathered}
\bar{A}_{i} \vee \bar{A}_{i+1} \vee \cdots \vee \bar{A}_{k}=\bar{A}_{i} \vee \bar{A}_{k}, \quad \text { for } 1 \leq i<k \leq t, \\
r_{M}\left(\bar{A}_{1} \cap \bar{A}_{2} \cap \cdots \cap \bar{A}_{t}\right)+\sum_{i=1}^{t-1} r_{M}\left(\bar{A}_{i} \cup \bar{A}_{i+1}\right) \leq \sum_{i=1}^{t} r_{M}\left(\bar{A}_{i}\right) .
\end{gathered}
$$

Since $\bar{A}_{j}=\operatorname{cl}_{M}\left(A_{j}\right)$ and since the join $A \vee B$ in $\mathcal{Z}(M)$ is $\operatorname{cl}_{M}(A \cup B)$, by equation (2) $A_{i} \cup A_{i+1} \cup \cdots \cup A_{k}$ and $A_{i} \cup A_{k}$ have the same closure in $M$, and so in $M / x$; thus, 
as needed, $A_{i} \vee A_{i+1} \vee \cdots \vee A_{k}=A_{i} \vee A_{k}$ in $\mathcal{Z}(M / x)$. The rank inequality in $M / x$ is immediate if $x$ is a loop of $M$, so assume this is not the case. Assume $x$ is in exactly $h$ of the cyclic flats $\bar{A}_{1}, \bar{A}_{2}, \ldots, \bar{A}_{t}$ of $M$. Thus,

$$
h+\sum_{i=1}^{t} r_{M / x}\left(A_{i}\right)=\sum_{i=1}^{t} r_{M}\left(\bar{A}_{i}\right) .
$$

That $x$ must be in at least $h$ of the sets $\bar{A}_{1} \cap \bar{A}_{2} \cap \cdots \cap \bar{A}_{t}$ and $\bar{A}_{i} \cup \bar{A}_{i+1}$ gives

$$
\begin{aligned}
h+r_{M / x}\left(A_{1} \cap A_{2} \cap\right. & \left.\cdots \cap A_{t}\right)+\sum_{i=1}^{t-1} r_{M / x}\left(A_{i} \cup A_{i+1}\right) \\
& \leq r_{M}\left(\bar{A}_{1} \cap \bar{A}_{2} \cap \cdots \cap \bar{A}_{t}\right)+\sum_{i=1}^{t-1} r_{M}\left(\bar{A}_{i} \cup \bar{A}_{i+1}\right) .
\end{aligned}
$$

The last two conclusions and inequality (3) give the counterpart of inequality (3) in $M / x$ for $A_{1}, A_{2}, \ldots, A_{t}$, as needed.

We omit the (similar) proof for $\mathcal{Z}(M \backslash x)$. (Deletions of transversal matroids are transversal, so only the result about $\mathcal{Z}(M / x)$ is needed to prove Theorem 3.2.)

The lemmas above complete the proof of Theorem 3.2.

Corollary 3.7 If each sublattice of $\mathcal{Z}(M)$ that is generated by an antichain of $\mathcal{Z}(M)$ is distributive and has dimension at most two, then $M$ and all of its minors, as well as their duals, are transversal.

Proof. Recall that $\mathcal{Z}\left(M^{*}\right)$ is isomorphic to the order dual of $\mathcal{Z}(M)$, so $M^{*}$ satisfies the hypotheses if and only if $M$ does. Thus, it suffices to show that for a lattice $L$, if each sublattice that is generated by an antichain is distributive and has dimension at most two, then $L$ is MI-orderable. Let $\mathcal{A}$ be an antichain of $L$. View the sublattice $\mathcal{A}$ generates as a suborder of $\mathbb{N}^{2}$ and list the elements of $\mathcal{A}$ as $a_{1}, a_{2}, \ldots, a_{t}$ where $a_{i}=\left(x_{i}, y_{i}\right)$ with $x_{1}>x_{2}>\cdots>x_{t}$; thus, $y_{1}<y_{2}<\cdots<y_{t}$. Clearly $a_{i} \vee a_{i+1} \vee \cdots \vee a_{k} \geq a_{i} \vee a_{k}$. Let $a_{i} \vee a_{k}$ be $(p, q)$. Thus, $p \geq x_{i}$ and $q \geq y_{k}$, so $(p, q) \geq\left(x_{j}, y_{j}\right)$ for $i \leq j \leq k$, and so $a_{i} \vee a_{i+1} \vee \cdots \vee a_{k}=a_{i} \vee a_{k}$, as needed. By the distributive law, property (ii) of Definition 3.1 can be written as $\left(a_{1} \vee a_{k+1}\right) \wedge\left(a_{2} \vee a_{k+1}\right) \wedge \cdots \wedge\left(a_{k} \vee a_{k+1}\right)=a_{k} \vee a_{k+1}$; this holds since $a_{i} \vee a_{k+1}=a_{i} \vee a_{i+1} \vee \cdots \vee a_{k} \vee a_{k+1} \geq a_{k} \vee a_{k+1}$ for $1 \leq i \leq k$.

We close this section by noting that if $N$ is a minor of $M$ and $\mathcal{Z}(M)$ is MI-orderable, $\mathcal{Z}(N)$ need not be MI-orderable; indeed, $\mathcal{Z}(N)$ might not even be a Tr-lattice. Consider the matroid $M$ in Figure 1. The isomorphic lattices $\mathcal{Z}(M)$ and $\mathcal{Z}\left(M^{*}\right)$ are MI-orderable. A direct check (or Theorem 5.1) shows that $\mathcal{Z}(M / x)$ (also shown in Figure 1) is MIorderable. However, by Theorem 4.1, its order dual, $\mathcal{Z}\left(M^{*} \backslash x\right)$, is not a Tr-lattice. This example also shows that the minor-closed classes of matroids described in Theorems 3.2 and Corollary 3.7 are not determined by lattice-theoretic properties that apply to the lattices of cyclic flats of all matroids in these classes. 


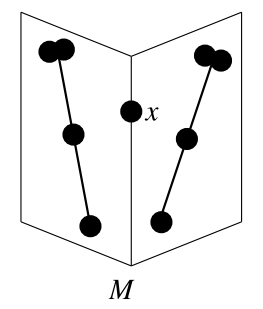

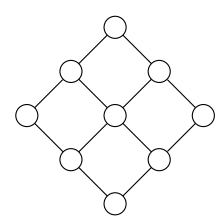

$Z(M)$

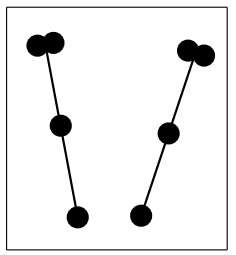

$M / x$

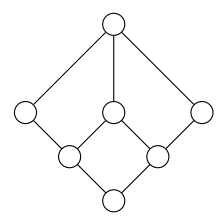

$Z(M / x)$

Figure 1: The lattice of cyclic flats of a matroid $M$ and that of $M / x$.

\section{A necessary condition for a lattice to be a Tr-lattice}

Property (ii) in Definition 3.1 forces each element of an MI-orderable lattice to have no more than two covers. We now show that the same is true of any Tr-lattice.

Theorem 4.1 Each element of a Tr-lattice has at most two covers.

Proof. Let the element $x$ of a lattice $L$ have at least three covers. We prove that $L$ is not a Tr-lattice by defining a function $\rho: L \rightarrow \mathbb{Z}$ so that properties (a)-(c) in Proposition 2.1 hold and inequality (MI) fails. For $y \in L$, let $F_{y}$ be the principal filter $\{u: u \geq y\}$ in $L$. Thus, the sublattice $F_{x}$ of $L$ has at least three atoms.

Define $\rho^{\prime}: L \rightarrow \mathbb{Z}$ by $\rho^{\prime}(y)=\left|L-F_{y}\right|$. It follows easily that $\rho^{\prime}$ satisfies properties (a)-(c) in Proposition 2.1. For $u, v, w \in F_{x}-\{x\}$, let

$m(u, v, w)=\rho^{\prime}(u)+\rho^{\prime}(v)+\rho^{\prime}(w)-\rho^{\prime}(u \vee v)-\rho^{\prime}(u \vee w)-\rho^{\prime}(v \vee w)+\rho^{\prime}(u \vee v \vee w)-\rho^{\prime}(x)$.

By inclusion-exclusion, $m(u, v, w)=\left|F_{x}-\left(F_{u} \cup F_{v} \cup F_{w}\right)\right|$. Set

$$
k=\min \{m(u, v, w): u, v, w>x\}=\left|F_{x}\right|-\max \left\{\left|F_{u} \cup F_{v} \cup F_{w}\right|: u, v, w>x\right\} .
$$

Thus, $k$ is the minimal size of the complement, in $F_{x}$, of the union of three proper principal filters in $F_{x}$. Note that if $k=m(u, v, w)$, then $u, v, w$ are distinct covers of $x$. Define $\rho: L \rightarrow \mathbb{Z}$ by

$$
\rho(y)= \begin{cases}\rho^{\prime}(y), & \text { if } y \leq x, \\ \rho^{\prime}(y)-k-1, & \text { otherwise }\end{cases}
$$

Clearly $\rho$ satisfies property (a) of Proposition 2.1. Properties (b) and (c) for $\rho$ follow from these properties for $\rho^{\prime}$ except in two cases:

(i) $\rho(y)<\rho(z)$ if $y<z, y \leq x$, and $z \not \leq x$, and

(ii) $\rho(y)+\rho(z) \geq \rho(y \vee z)+\rho(y \wedge z)$ if $y \not \leq x, z \not \leq x$, and $y \wedge z \leq x$. 


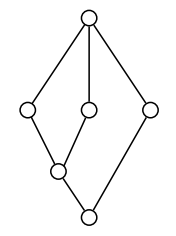

(a)

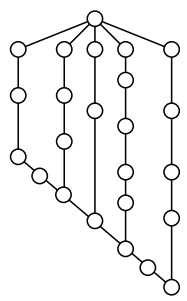

(b)

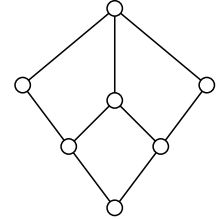

(c)

Figure 2: (a) The lattice $L_{8}$. (b) An example of the generalization of $L_{8}$. (c) A lattice $L_{I}$ obtained from an ideal in a product of two three-element chains.

Similar arguments apply in these cases, so we address just the second. Thus, assume $y \not \leq x, z \not \leq x$, and $y \wedge z \leq x$. The inequality in statement (ii), when simplified, is $\left|F_{y \wedge z}-\left(F_{y} \cup F_{z}\right)\right| \geq k+1$. That $\left(F_{y} \cup F_{z}\right) \cap F_{x}$ is the union of two principal filters, $F_{y \vee x}$ and $F_{z \vee x}$, both properly contained in $F_{x}$, gives $\left|F_{x}-\left(F_{y} \cup F_{z}\right)\right| \geq k+1$; the required inequality follows since $F_{x} \subseteq F_{y \wedge z}$.

Let $M$ be a matroid arising from $L$ and $\rho$ as in Proposition 2.1. Fix $u, v, w$ with $k=m(u, v, w)$ and let $U, V$, and $W$ be the corresponding cyclic flats of $M$. The definitions of $m$ and $\rho$ give

$$
r(U)+r(V)+r(W)-r(U \cup V)-r(U \cup W)-r(V \cup W)+r(U \cup V \cup W)=r(X)-1 .
$$

Since $r(X) \leq r(U \cap V \cap W)$, it follows that the antichain $\{U, V, W\}$ of $\mathcal{Z}(M)$ does not satisfy inequality (MI). Thus, $M$ is not transversal, so $L$ is not a Tr-lattice.

A matroid $M$ is nested if $\mathcal{Z}(M)$ is a chain. These matroids have arisen many times in a variety of contexts (see [3, Section 4] for more information). That $\mathcal{Z}(M \oplus N)$ is isomorphic to the product $\mathcal{Z}(M) \times \mathcal{Z}(N)$ gives the following corollary.

Corollary 4.2 If $\mathcal{Z}(M)$ is a Tr-lattice, then the matroid obtained from $M$ by deleting all loops and isthmuses is either a direct sum of at most two nested matroids or it is connected.

\section{$5 \quad$ Examples and constructions}

Acketa [2] proved that the lattice $L_{8}$ in Figure 2(a) is a Tr-lattice but its order dual is not. (This and the other results and conjectures in $[1,2]$ are easily addressed by the results above.) Note that $L_{8}$ and the lattice in Figure 2(b) are in an infinite family of MI-orderable lattices, the defining properties of which are that the interval between $\hat{0}$ and any coatom is a chain, and for one of these chains (e.g., the left-most chain in Figure 2(b)), all other such chains intersect it in different initial segments.

Sublattices of MI-orderable lattices are clearly MI-orderable. The next result gives another simple construction for MI-orderable lattices. (See Figure 2(c).) 

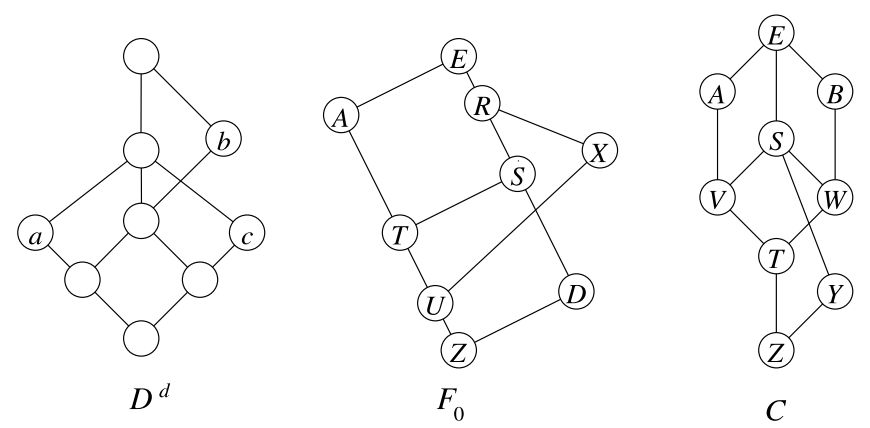

Figure 3: Three nonplanar Tr-lattices; only $D^{d}$ is MI-orderable.

Theorem 5.1 For any ideal I in an MI-orderable lattice L, the lattice $L_{I}$ induced on the set $I \cup\{\hat{1}\}$ by the same order is MI-orderable.

Proof. Each antichain $\mathcal{A}$ of $L_{I}$ is an antichain of $L$; order $\mathcal{A}$ so that properties (i) and (ii) of Definition 3.1 hold in $L$. Let $z$ be the join of $\left\{a_{i}, a_{i+1}, \ldots, a_{k}\right\}$ and of $\left\{a_{i}, a_{k}\right\}$ in $L$. If $z \in I$, then $z$ is the join of each of these sets in $L_{I}$, otherwise both sets have join $\hat{1}$ in $L_{I}$. Thus, property (i) holds in $L_{I}$. The same ideas show that property (ii) holds in $L_{I}$ since the meet operations are identical in $L$ and $L_{I}$.

Recall that the linear (or ordinal) sum of partial orders $P$ and $Q$, where $P$ and $Q$ are disjoint, is the order on $P \cup Q$ in which the restriction to $P$ (resp., $Q$ ) is the order on $P$ (resp., $Q$ ) and all elements of $P$ are less than all elements of $Q$. Note that the class of MI-orderable lattices is closed under linear sums and under the closely-related operation that, given lattices $L$ and $L^{\prime}$, forms the Hasse diagram of the new lattice from those of $L$ and $L^{\prime}$ by identifying the greatest element of $L$ with the least element of $L^{\prime}$. It follows from Theorem 5.4 below that the same results hold for Tr-lattices. By Theorem 4.1, the class of MI-orderable lattices and that of Tr-lattices are not closed under direct products.

We next treat three particular Tr-lattices of dimension 3, only one of which is MIorderable. These lattices, which are shown in Figure 3, are among the forbidden sublattices for planar lattices (see [6]). (No other forbidden sublattices for planar lattices satisfy the necessary condition for Tr-lattices given in Theorem 4.1.)

Theorem 5.2 The lattice $D^{d}$ is MI-orderable. The lattices $F_{0}$ and $C$ are Tr-lattices but are not MI-orderable.

Proof. Note that each antichain with one or two elements is MI-orderable. From this remark, the MI-orderable lattice in Figure 2(c), and the MI-ordering $b, a, c$ of the antichain $\{a, b, c\}$ of $D^{d}$, it follows that $D^{d}$ is MI-orderable.

In the lattice $F_{0}$, the only antichains with more than two elements are $\{A, S, X\}$, $\{X, T, D\}$, and $\{X, A, D\}$. The first two are MI-orderable (ordered as written), so we need only show that in any matroid $M$ for which $\mathcal{Z}(M)$ is isomorphic to $F_{0}$, the flats that 
correspond to $X, A, D$ (for which we use the same notation) satisfy inequality (MI), that is, $r(X)+r(A)+r(D)-r(R)-r(E) \geq r(X \cap A \cap D)$. By semimodularity,

$$
r(A)+r(S) \geq r(E)+r(A \cap S) .
$$

The inclusions $T \subseteq A \cap S \subseteq S$ give $\operatorname{cl}((A \cap S) \cup X)=R$, so

$$
r(A \cap S)+r(X) \geq r(R)+r(A \cap S \cap X) .
$$

The inclusions $U \subseteq A \cap S \cap X \subseteq S$ give $\operatorname{cl}((A \cap S \cap X) \cup D)=S$, so

$$
r(A \cap S \cap X)+r(D) \geq r(S)+r(A \cap S \cap X \cap D) .
$$

Note that $A \cap S \cap X \cap D$ is $A \cap X \cap D$. Adding the three inequalities and simplifying yields the desired inequality.

A similar argument applies to the lattice $C$, for which it suffices to consider the antichains $\{A, B, Y\},\{A, W, Y\},\{B, V, Y\}$, and $\{V, W, Y\}$. The last three are listed in MI-orderings. For $\{A, B, Y\}$, apply semimodularity to the pairs $\{A, S\},\{A \cap S, B\}$, and $\{A \cap S \cap B, Y\}$; the inclusions $V \subseteq A \cap S \subseteq S$ and $T \subseteq A \cap S \cap B \subseteq S$ give $\operatorname{cl}((A \cap S) \cup B)=E$ and $\operatorname{cl}((A \cap S \cap B) \cup Y)=S$; add the resulting inequalities and cancel the common terms to get inequality (MI) for $\{A, B, Y\}$.

We now consider two operations for producing new Tr-lattices. Given lattices $L_{1}$ and $L_{2}$, let $L_{1} * L_{2}$ be the lattice on $\left(L_{1} \cup L_{2} \cup\{\hat{0}, \hat{1}\}\right)-\left\{\hat{1}_{L_{1}}, \hat{1}_{L_{2}}\right\}$ with $x \leq y$ if and only if (i) $y=\hat{1}$, or (ii) $x=\hat{0}$, or (iii) for some $i \in\{1,2\}$, both $x$ and $y$ are in $L_{i}$ and $x \leq y$ in $L_{i}$. Figure 4(a) illustrates this operation; note that the unique four-element antichain in this lattice is not MI-orderable.

Theorem 5.3 If $L_{1}$ and $L_{2}$ are Tr-lattices, then so is $L_{1} * L_{2}$.

The proofs of Theorems 5.3 and 5.4 are similar, so we prove only the latter, which concerns lexicographic sums [10, Section 1.10]. Let $L$ be a lattice and let $\mathcal{L}=\left(L_{x}: x \in L\right)$ be a family of lattices that is indexed by the elements of $L$. The lexicographic sum $L \oplus \mathcal{L}$ is defined on the set $\left\{(x, a): x \in L, a \in L_{x}\right\}$; the order is given by $(x, a) \leq(y, b)$ if and only if either (i) $x<y$ in $L$ or (ii) $x=y$ and $a \leq b$ in $L_{x}$. Figure 4 (b) illustrates this operation. It is easy to see that $L \oplus \mathcal{L}$ is not necessarily MI-orderable even if all of the constituent lattices are.

Theorem 5.4 If $L$ has width at most two and if $\mathcal{L}=\left(L_{x}: x \in L\right)$ is a family of Tr-lattices, then $L \oplus \mathcal{L}$ is a Tr-lattice.

Proof. Let $\phi: \mathcal{Z}(M) \rightarrow L \oplus \mathcal{L}$ be an isomorphism. We must show that any antichain $\mathcal{A}$ in $\mathcal{Z}(M)$ satisfies inequality (MI).

For $F \in \mathcal{Z}(M)$, let $\phi_{1}(F)$ be the first component of $\phi(F)$; thus, $\phi_{1}(F) \in L$. For $x \in \phi_{1}(\mathcal{A})$, set $\mathcal{A}_{x}=\left\{F: F \in \mathcal{A}, \phi_{1}(F)=x\right\}$. Since $L$ has width at most two and $\mathcal{A}$ is an antichain in $\mathcal{Z}(M)$, there are at most two such sets; these sets partition $\mathcal{A}$. 


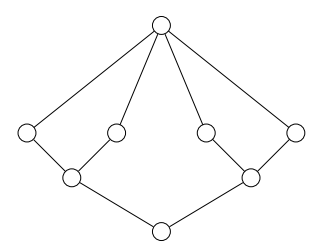

(a)

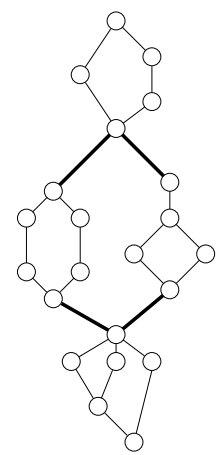

(b)

Figure 4: (a) The lattice $L_{1} * L_{2}$ where $L_{1}$ and $L_{2}$ are Boolean lattices on two elements. (b) A lexicographic sum; the indexing lattice is a Boolean lattice on two elements.

For $u \in L$, let $Z_{u}$ and $E_{u}$ be the least and greatest flats $F \in \mathcal{Z}(M)$ with $\phi_{1}(F)=u$. Thus, if $\phi_{1}(A)=u$ and $\phi_{1}(B)=v$ with $u \neq v$, then $A \vee B=Z_{u \vee v}$ and $A \wedge B=E_{u \wedge v}$ by the definition of $L \oplus \mathcal{L}$.

Let $x$ be in $\phi_{1}(\mathcal{A})$. Note that $\mathcal{Z}\left(M \mid E_{x} / Z_{x}\right)$ is isomorphic to $L_{x}$, so $M \mid E_{x} / Z_{x}$ is transversal. Applying Proposition 2.2 to the antichain $\left\{A-Z_{x}: A \in \mathcal{A}_{x}\right\}$ of $\mathcal{Z}\left(M \mid E_{x} / Z_{x}\right)$, writing the resulting inequality in terms of the rank function of $M$, and simplifying gives

$$
r\left(\bigcap\left(\mathcal{A}_{x}\right)\right) \leq \sum_{\mathcal{F} \subseteq \mathcal{A}_{x}}(-1)^{|\mathcal{F}|+1} r(\bigcup(\mathcal{F}))
$$

This is inequality $(\mathrm{MI})$ for $\mathcal{A}$ if $\left|\phi_{1}(\mathcal{A})\right|=1$. Assume, instead, $\phi_{1}(\mathcal{A})=\{x, y\}$. The counterpart of inequality (4) holds with $\mathcal{A}_{y}$ in place of $\mathcal{A}_{x}$. Since $r(X \cup Y)=r\left(Z_{x \vee y}\right)$ for any $X \in L_{x}$ and $Y \in L_{y}$,

$$
\begin{aligned}
& \sum_{\mathcal{F} \subseteq \mathcal{A}}(-1)^{|\mathcal{F}|+1} r(\bigcup(\mathcal{F}))=\sum_{\mathcal{F} \subseteq \mathcal{A}_{x}}(-1)^{|\mathcal{F}|+1} r(\bigcup(\mathcal{F}))+\sum_{\mathcal{F} \subseteq \mathcal{A}_{y}}(-1)^{|\mathcal{F}|+1} r(\bigcup(\mathcal{F})) \\
& +\sum_{\substack{\mathcal{F}_{x} \subseteq \mathcal{A}_{x}, \mathcal{F}_{x} \neq \emptyset \\
\mathcal{F}_{y} \subseteq \mathcal{A}_{y}, \mathcal{F}_{y} \neq \emptyset}}(-1)^{\left|\mathcal{F}_{x}\right|+\left|\mathcal{F}_{y}\right|+1} r\left(\bigcup\left(\mathcal{F}_{x}\right) \cup \bigcup\left(\mathcal{F}_{y}\right)\right) \\
& =\sum_{\mathcal{F} \subseteq \mathcal{A}_{x}}(-1)^{|\mathcal{F}|+1} r(\bigcup(\mathcal{F}))+\sum_{\mathcal{F} \subseteq \mathcal{A}_{y}}(-1)^{|\mathcal{F}|+1} r(\bigcup(\mathcal{F})) \\
& -r\left(Z_{x \vee y}\right) \sum_{\mathcal{F}_{x} \subseteq \mathcal{A}_{x}, \mathcal{F}_{x} \neq \emptyset}(-1)^{\left|\mathcal{F}_{x}\right|} \sum_{\mathcal{F}_{y} \subseteq \mathcal{A}_{y}, \mathcal{F}_{y} \neq \emptyset}(-1)^{\left|\mathcal{F}_{y}\right|},
\end{aligned}
$$

so inequality (4) and semimodularity give

$$
\sum_{\mathcal{F} \subseteq \mathcal{A}}(-1)^{|\mathcal{F}|+1} r(\bigcup(\mathcal{F})) \geq r\left(\bigcap\left(\mathcal{A}_{x}\right)\right)+r\left(\bigcap\left(\mathcal{A}_{y}\right)\right)-r\left(Z_{x \vee y}\right) \geq r(\bigcap(\mathcal{A})) .
$$


Thus, inequality (MI) holds, as needed.

We close by noting that, by duality, if $L$ has width at most two and if $\mathcal{L}=\left(L_{x}: x \in L\right)$ is a family of bi-transversal lattices (i.e., all lattices in $\mathcal{L}$ and their order duals are Trlattices), then $L \oplus \mathcal{L}$ is bi-transversal.

\section{ACKNOWLEDGEMENTS}

I am very grateful for Joseph Kung, whose questions and comments prompted me to pursue more deeply the implications of Proposition 2.2. I thank the referee for reading the manuscript carefully, providing useful comments, and raising the point that led to the closing remark.

\section{References}

[1] D. Acketa, On the essential chains and squares, in Finite and Infinite Sets, Vol. I, II, A. Hajnal, L. Lovász, and V. T. Sós, eds., (North-Holland, Amsterdam, 1984) 25-33.

[2] D. Acketa, The inverse of a Tr-lattice need not be a Tr-lattice, Univ. u Novom Sadu Zb. Rad. Prirod.-Mat. Fak. Ser. Mat. 13 (1983) 317-338.

[3] J. Bonin and A. de Mier, Lattice path matroids: structural properties, European J. Combin. 27 (2006) 701-738.

[4] J. Bonin and A. de Mier, The lattice of cyclic flats of a matroid, Ann. Comb., to appear.

[5] A. W. Ingleton, Transversal matroids and related structures, in: Higher Combinatorics, (Reidel, Dordrecht, 1977) 117-131.

[6] D. Kelly and I. Rival, Planar lattices, Canad. J. Math. 27 (1975) 636-665.

[7] J. G. Oxley, Matroid Theory, (Oxford University Press, Oxford, 1992).

[8] J. A. Sims, An extension of Dilworth's theorem, J. London Math. Soc. 16 (1977) 393-396.

[9] J. A. Sims, Some Problems in Matroid Theory, (Ph.D. Dissertation, Linacre College, Oxford University, Oxford, 1980).

[10] W. T. Trotter, Combinatorics and Partially Ordered Sets: Dimension Theory, (The Johns Hopkins University Press, Baltimore, 1992). 